\title{
Five-long non-coding RNA risk score system for the effective prediction of gastric cancer patient survival
}

\author{
ZUNQI HU*, DEJUN YANG ${ }^{*}$, YUAN TANG, XIN ZHANG, ZIRAN WEI, \\ HONGBING FU, JIAPENG XU, ZHENXIN ZHU and QINGPING CAI \\ Department of Gastrointestinal Surgery, Changzheng Hospital, \\ Second Military Medical University, Shanghai 200003, P.R. China
}

Received May 1, 2018; Accepted December 12, 2018

DOI: $10.3892 / 01.2019 .10124$

\begin{abstract}
The prognosis for patients with gastric cancer (GC) is usually poor, as the majority of patients have reached the advanced stages of disease at the point of diagnosis. Therefore, revealing the mechanisms of GC is necessary for the identification of key biomarkers and the development of effective targeted therapies. The present study aimed to identify long non-coding RNAs (lncRNAs) prominently expressed in patients with GC. The GC dataset (including 384 GC samples) was downloaded from The Cancer Genome Atlas database as the training set. A number of other GC datasets were obtained from the Gene Expression Omnibus database as validation sets. Following data processing, lncRNAs were annotated, followed by co-expression module analysis to identify stable modules, using the weighted gene co-expression network analysis (WGCNA) package. Prognosis-associated lncRNAs were screened using the 'survival' package. Following the selection of the optimal lncRNA combinations using the 'penalized' package, risk score systems were constructed and assessed. Consensus differentially-expressed RNAs (DE-RNAs) were screened using the MetaDE package, and an IncRNA-mRNA network was constructed. Additionally, pathway enrichment analysis was conducted for the network nodes using gene set enrichment analysis (GSEA). A total of seven modules (blue, brown, green, grey, red, turquoise and yellow) were obtained following WGCNA analysis, among which the green and turquoise modules were stable and associated with the histological grade of GC. A total of 12 prognosis-associated
\end{abstract}

Correspondence to: Dr Zhenxin Zhu or Dr Qingping Cai, Department of Gastrointestinal Surgery, Changzheng Hospital, Second Military Medical University, 415 Fengyang Road, Shanghai 200003, P.R. China

E-mail:drzzx@163.com

E-mail: caiqingping@smmu.edu.cn

${ }^{*}$ Contributed equally

Key words: gastric cancer, long non-coding RNAs, weighed gene co-expression network analysis, survival curve, risk score system
lncRNAs were identified in the two modules. Combined with the optimal lncRNA combinations, risk score systems were constructed. The risk score system based on the green module [including ITPK1 antisense RNA 1 (ITPK1-AS1), KCNQ1 downstream neighbor (KCNQ1DN), long intergenic non-protein coding RNA 167 (LINC00167), LINC00173 and LINC00307] was the more efficient at predicting risk compared with those based on the turquoise, or the green + turquoise modules. A total of 1,105 consensus DE-RNAs were identified; GSEA revealed that LINC00167, LINC00173 and LINC00307 had the same association directions with 4 pathways and the 32 genes involved in those pathways. In conclusion, a risk score system based on the green module may be applied to predict the survival of patients with GC. Furthermore, ITPKI-AS1, KCNQIDN, LINC00167, LINC00173 and LINC00307 may serve as biomarkers for $\mathrm{GC}$ pathogenesis.

\section{Introduction}

Gastric cancer (GC) originates from the lining of the stomach, and may metastasize to other tissues and organs, including the lungs, liver, lymph nodes and bones (1). It is estimated that 22,220 new cases of GC were diagnosed and 10,990 patients succumbed to GC in 2014 (2). GC is more common in males, and has a high incidence in East Asia and Eastern Europe (3). The most common inducer of GC is Helicobacter pylori infection, although other risk factors include pickled foods, smoking and obesity $(4,5)$. Patients with GC usually have an unfavorable prognosis, as the majority reach the advanced stages of disease prior to diagnosis (6). Therefore, determining the mechanisms of $\mathrm{GC}$ is required for the identification of key biomarkers and the development of effective targeted therapies.

The human genome project indicates that only $1.2 \%$ of the mammalian genome encodes proteins (7), and that the majority of the genome is transcribed to tens of thousands of long non-coding RNAs (lncRNAs), which are $>200 \mathrm{nt}$ in length (8). IncRNAs function in various biological processes, including cellular development and differentiation (9). It has increasingly been suggested that the principal role of lncRNAs is the guidance of site specificity for chromatin-modifying complexes in order to effect epigenetic alterations (10). lncRNAs act through a number of mechanisms in the control of cancer. For instance, specific lncRNAs are key regulators of 
the protein signaling pathways underlying carcinogenesis (11). Additionally, other lncRNAs function as decoys, sequestering biomolecules and preventing cancerous cells from fulfilling their cellular roles $(12,13)$. Numerous studies have reported the important role of lncRNAs in GC; 1 ncRNA-H19 is upregulated in $\mathrm{GC}$ tissues and affects the progression and metastasis of GC by promoting isthmin 1 expression and inhibiting calneuron 1 expression (14). The downregulated expression of lncRNA maternally expressed gene 3 promotes cell proliferation and apoptosis, and predicts a poor prognosis in GC $(15,16)$. Overexpression of the lncRNA colon cancer associated transcript 2 associates with the progression of GC and may serve as a promising prognostic marker for the disease (17). Antisense ncRNA in the INK4 locus acts as a growth regulator in GC by silencing microRNA ( $m i R)-99 a$ and $m i R-449 a$, and may indicate a potential prognostic biomarker and therapeutic target in GC $(18,19)$. BRAF-activated non-coding RNA overexpression associates positively with tumor depth, clinical stage and tumor metastasis, and predicts a poor prognosis in patients with GC (20). However, the functions of numerous IncRNAs remain unclear; therefore, it is necessary to conduct a comprehensive assessment of the functions of lncRNAs in GC.

Bioinformatics analysis of gene expression profiles has been widely applied to investigate the pathogenesis of various diseases (21). In the current study, multiple GC datasets were searched and downloaded from open access databases. Using comprehensive bioinformatics analyses, certain prognosis-associated lncRNAs were identified. An optimal risk score system based on these IncRNAs was constructed to evaluate the risk of developing GC, the efficiency of which was determined using various independent datasets.

\section{Subjects and methods}

Data sources. The mRNA-sequencing data for GC, sequenced on the Illumina HiSeq 2000 RNA Sequencing platform (Illumina, Inc., San Diego, CA, USA), were downloaded from The Cancer Genome Atlas (TCGA; https://cancergenome.nih. gov/) database, which included 384 GC samples. Among the 384 samples, there were 122 samples from deceased patients due to GC, 238 samples from surviving patients (mean survival time, mean \pm standard deviation, $16.17 \pm 16.96$ months) and 24 samples without survival information.

From the Gene Expression Omnibus (GEO, http://www. ncbi.nlm.nih.gov/geo/) database, three kinds of datasets (dataset I-III) were searched and identified using 'gastric cancer' as the key words. Dataset I was searched according to the following criteria: i) The dataset was a gene expression profile; ii) the samples were tumor tissues from patients with GC; iii) the dataset was a human expression profile; and iv) the total number of samples was $\geq 100$. Thus, GSE15459 (22-26) (including 300 GC samples) and GSE54129 (including 111 GC samples) sequenced on the Affymetrix-GPL570 platform (Affymetrix; Thermo Fisher Scientific, Inc., Waltham, MA, USA) were selected. The criteria for searching GC dataset II were as follows: i) The dataset was a gene expression profile; ii) the samples were tumor tissues from patients with GC; iii) the dataset was a human expression profile; iv) the samples contained survival information; and v) the total number of samples was $\geq 100$. Only GSE62254 (27) (including 300 GC samples; Affymetrix-GPL570 platform; Affymetrix; Thermo Fisher Scientific, Inc.,) was selected, involving 135 samples from deceased patients due to GC, and 148 samples from surviving patients (mean survival time, mean \pm standard deviation, 50.59 \pm 31.42 months), and 17 samples without survival information. Dataset III was selected according to the following criteria: i) The dataset was a gene expression profile; ii) the samples were tumor tissues from patients with GC; iii) there were control tissues; iv) the dataset was a human expression profile; and v) the total number of samples was $\geq 50$. Ultimately, the GSE65801 (28) (including 32 GC samples and 32 control samples; Agilent GPL14550 platform; Agilent Technologies, Inc., Santa Clara, CA, USA), GSE29998 (29) (including 50 GC samples and 49 control samples; GPL6947 Illumina HumanHT-12 V3.0 platform; Illumina, Inc.), GSE33335 (30-32) (including 25 GC samples and 25 control samples; GPL5175 [HuEx-1_0-st] platform) and GSE27342 $(33,34)$ (including $80 \mathrm{GC}$ samples and 80 control samples; GPL5175 [HuEx-1_0-st] platform) datasets were selected.

Data preprocessing. The data from the aforementioned databases were divided into three types based on the testing platforms. For the dataset from TCGA, the quantile standardization method in the $\mathrm{R}$ package preprocessCore (version 1.40.0; http://bioconductor.org/packages/release/bioc/html/preprocessCore. html) (35) was used for data normalization. For the CIMFast Event Language data sequenced on the Affymetrix platform, the $\mathrm{R}$ package oligo (version 1.41.1; http://www.bioconductor.org/packages/release/bioc/html/oligo.html) (36) was utilized for format conversion, missing data filling, background correction and data normalization. For the TXT data sequenced on the Agilent platform, the R package Limma (version 3.34.0; https://bioconductor. org/packages/release/bioc/html/limma.html) (37) was applied for $\log 2 \operatorname{logarithmics}$ and data normalization.

Subsequently, IncRNAs were annotated based on the Ref_seq and Transcript_ID provided by the annotation platform, and aligned with human genome sequences (version, GRCh38) on a platform using Clustal 2.1 software (http://www. clustal.org/clustal2/) (38). Subsequently, multiple annotation results were merged to identify the lncRNAs and their corresponding expression information (39-41).

Weighted gene co-expression network analysis (WGCNA) to identify disease-associated modules. As a bioinformatics algorithm for building co-expression networks, WGCNA is used to identify disease-associated modules and thus screen for pathogenic processes and potential therapeutic targets (42). In the present study, based on the use of TCGA dataset as the training dataset, and GSE15459 and GSE54129 as the validation datasets, stable modules associated with GC were identified using the R package WGCNA (version 1.61; https://cran.r-project.org/web/packages/WGCNA/index. html) (43). Expression correlation between every two of the three datasets was calculated, and the adjacent function was defined as follows: WGCNA analysis was required to satisfy the precondition of scale-free network distribution, and thus the value of the adjacency matrix weighting parameter 'power' was investigated. Based on the RNA data, the squares of the 
A



B

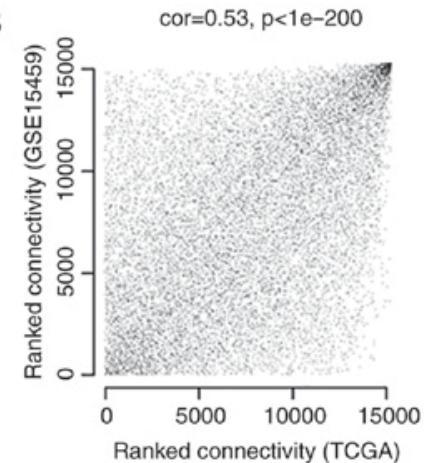

cor $=0.88, p<1 e-200$

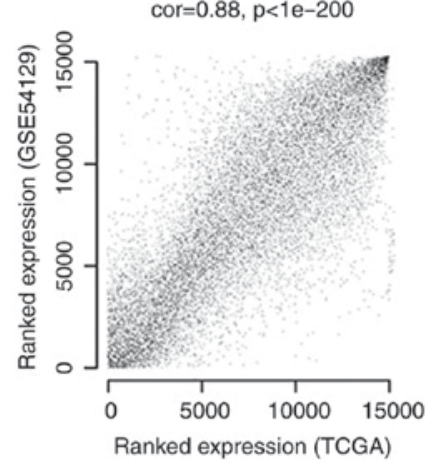

cor $=0.56, p<1 e-200$

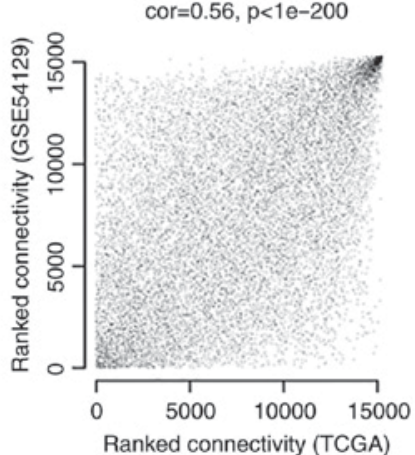

cor $=0.95, p<1 e-200$
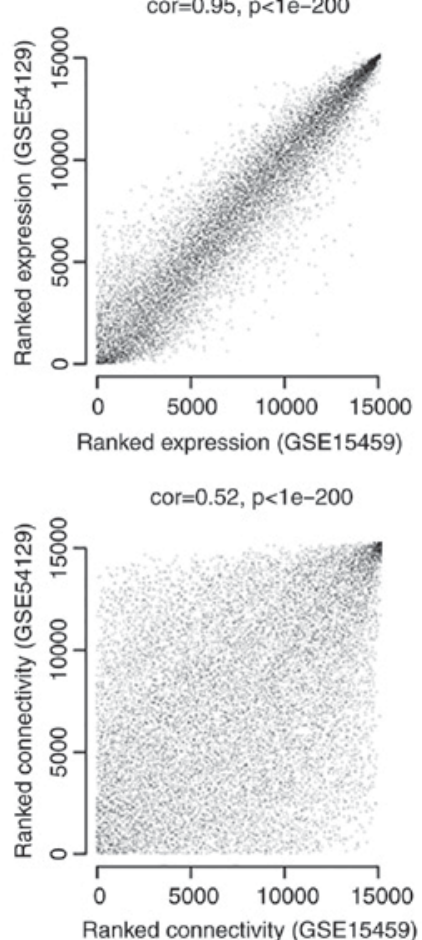

Figure 1. The correlation analysis of the datasets. (A) Expression correlations and (B) connection correlations between the RNAs of every two entries of TCGA dataset, GSE15459 and GSE54129. Left, middle, and right diagrams represent TCGA-GSE15459, TCGA-GSE54129 and GSE15459-GSE54129, respectively. TCGA, The Cancer Genome Atlas; GSE, gene set enrichment.

correlation coefficients between $\log (\mathrm{k})$ and $\log [\mathrm{p}(\mathrm{k})]$ were calculated for different 'power' values. A higher square value indicated that the network was closer to a scale-free network distribution. Following the definition of the adjacent function, module partition was conducted (the thresholds for module partition were that the module contained $\geq 150$ RNAs and a cutHeight of 0.99). Combined with the clinical information from TCGA dataset, the correlation between each module and the clinical information was analyzed. Functional annotation was conducted for each stable module using the userListEnrichment function in the WGCNA package (43). Additionally, differential expression analysis of lncRNAs between tumor and control groups was performed for each module, with a $\mathrm{P}$-value and false discovery rate (FDR) of $<0.05$.

Selection of prognosis-associated lncRNAs. Based on the lncRNAs obtained in the clinical factors-associated stable modules, univariate Cox regression analysis was performed using the R package survival (version 2.4; https://cran.r-project. org/web/packages/survival/index.html) (44) for the GC samples with survival information in the TCGA dataset, to identify prognosis-associated lncRNAs. A log-rank P-value of $<0.05$ was considered to indicate a statistically significant difference.

Construction and assessment of a risk score system. The lncRNAs in the stable modules that correlated significantly with notable clinical factors were analyzed separately. Using the Cox-Proportional Hazards (Cox-PH) model in the R package 'penalized' (http://bioconductor.org/packages/penalized/) (45), the optimal lncRNA combinations were selected. The parameter 'lambda' was obtained with
1,000 circulation calculations using a cross-validation likelihood algorithm (46). Subsequently, the risk score system was constructed, combined with the regression coefficient $(\beta)$ and expression level (expr $\operatorname{lncRNA}_{\text {nc }}$ ) of each lncRNA in the optimal lncRNA combination. The risk score of each sample was calculated using the following formula:

Risk score $=\beta_{\text {IncRNA1 }} \times \operatorname{expr}_{\text {lncRNA1 }}+\beta_{\text {lncRNA2 }} \times \operatorname{expr}_{\text {IncRNA2 }}+\cdots+$
$\beta_{\text {lncRNAn }} \times \operatorname{expr}_{\text {lncRNAn }}$.

The samples in the TCGA dataset were divided into high-risk and low-risk groups according to the median of their risk scores. Kaplan-Meier survival curves were used to evaluate the correlation between the overall survival of the samples and the two groups. Using GSE15459 and GSE54129 as the validation datasets, the robustness of the risk score system in predicting sample risk and prognosis was assessed. Moreover, the predictive results of the risk score system in the training and validation datasets were compared to identify the optimal model for subsequent analyses.

Differential expression analysis of notable lncRNAs in multiple datasets. Using the MetaDE.ES algorithm in the $\mathrm{R}$ package MetaDE (version 1.0.5; https://cran.r-project. org/web/packages/MetaDE/) (47,48), consensus differentially expressed RNAs (DE-RNAs; between GC and control samples) were screened from the GSE65801, GSE29998, GSE33335 and GSE27342 datasets. $\tau^{2}=0$, Qpval $>0.05, \mathrm{P}<0.05$ and FDR $<0.05$ were set as the cut-off criteria. The focus was the differential expression of the notable lncRNAs that were screened as disease or prognosis related-lncRNAs. 
A

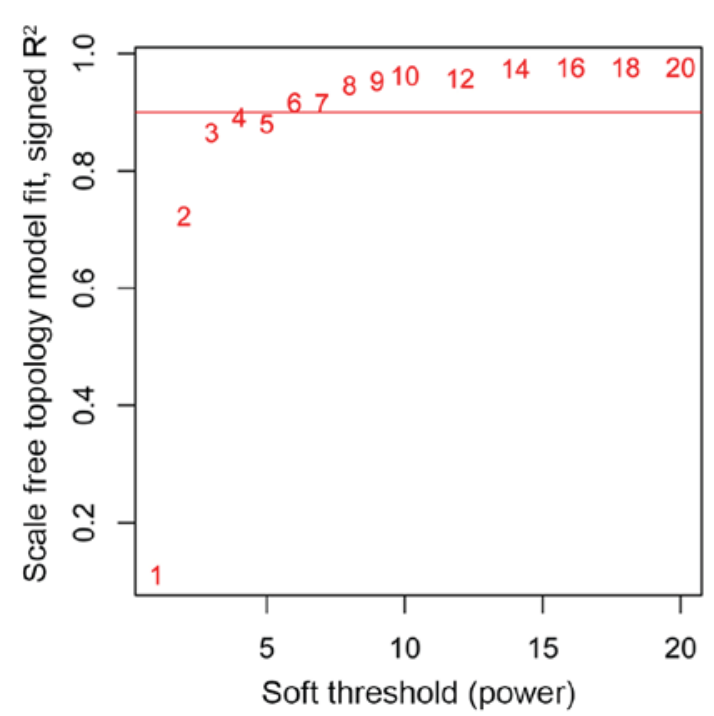

B

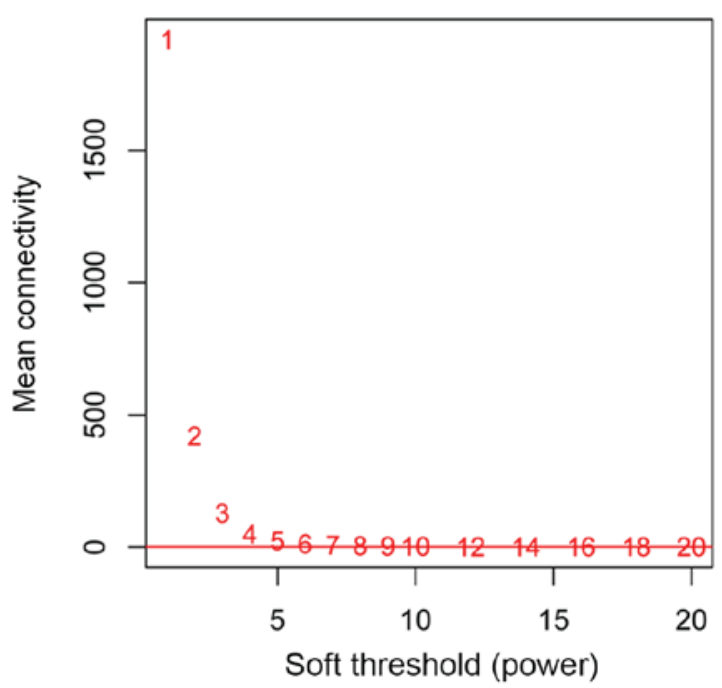

Figure 2. Selection diagram of the adjacency matrix weighting parameter 'power'. (A) The red line is the standard when the square of the correlation coefficient reaches 0.9 . (B) The mean connectivity of RNAs under different values of 'power' (the red line represents the mean connectivity of 2 when the power is 6 ).

Analysis of IncRNA-associated pathways. Based on the correlation coefficients between notable lncRNAs and mRNAs that were located in the same WGCNA module, the lncRNA-mRNA network was constructed. Subsequently, pathway enrichment analysis was conducted for the network nodes using Gene Set Enrichment Analysis (GSEA; http://software.broadinstitute. org/gsea/index.jsp) (49). A nominal P-value of $<0.05$ was considered to indicate a statistically significant difference.

\section{Results}

Identification of GC-associated stable modules based on WGCNA. Following data preprocessing, a total of 988 lncRNAs and 15,127 mRNAs shared by TCGA dataset, GSE15459 and GSE54129 were identified. TCGA dataset was taken as the training dataset, whilst GSE15459 and GSE54129 were used as the validation datasets to screen for GC-associated RNA modules.

To ensure that the RNA expression levels in each dataset were comparable, expression consistency analysis was performed for the expression values of shared RNAs. As outlined in Fig. 1A, the correlation in expression between every two of the three datasets was $>0.85$, and P-values were $<1 \times 10^{-200}$, indicating significant positive correlations between every two datasets and suggesting that these data sets are comparable and suitable for further analysis. Additionally, the correlations of connectivity between nodes were $>0.5$, and the $\mathrm{P}$-values were $<1 \times 10^{-200}$, suggesting that connection correlations between the RNAs of every two datasets were positive (Fig. 1B).

Following definition of the adjacent function, the power value of 6 , for which the square value of the correlation coefficient reached 0.9 for the first time, was selected (Fig. 2A). Under a power value of 6 , the mean connectivity degree of the RNAs was 2, which conformed to the small-world property in a scale-free network (Fig. 2B).
Following construction of the co-expression network (based on TCGA dataset), the stable modules associated with disease were screened. A total of seven modules (blue, brown, green, grey, red, turquoise and yellow) were obtained (Fig. 3A). The differentially expressed lncRNAs between the tumor and control groups in the seven modules are listed in Table I. Combined with the seven modules and the RNAs involved in each module, corresponding module partition was conducted in GSE15459 (Fig. 3B) and GSE54129 (Fig. 3C).

For TCGA dataset, the module partition and module correlations are presented in Fig. 4. The results illustrate that the RNAs in the same module tended to cluster together, including the green or blue nodes, indicating that the RNAs have more similar expression levels (Fig. 4A). The green and blue modules have the characteristics of independent branches (Fig. 4B).

The stabilities of the seven modules were assessed, and the blue, green, red, turquoise and yellow modules were deemed stable (preservation $\mathrm{Z}$ score $>5$ ). The top three modules were turquoise, green and yellow, according to the preservation $\mathrm{Z}$ score, and these three may be associated with GC pathogenesis. Functional annotation for each stable module revealed that the lncRNAs in the turquoise (including 46 lncRNAs), green (including 30 lncRNAs) and yellow (including 32 lncRNAs) modules were predominantly enriched in cell adhesion, immune response and digestion, respectively (Table II).

In addition, based on the clinical information in TCGA dataset, the correlation between each module and the clinical factors was analyzed. Among the 5 stable modules, the green and turquoise modules correlated significantly with histological grade (Fig. 4C). Therefore, the green and turquoise modules were further analyzed.

Selection of prognosis-associated lncRNAs. Based on the 76 IncRNAs in the green and turquoise modules, 12 prognosis-associated IncRNAs were identified in TCGA 

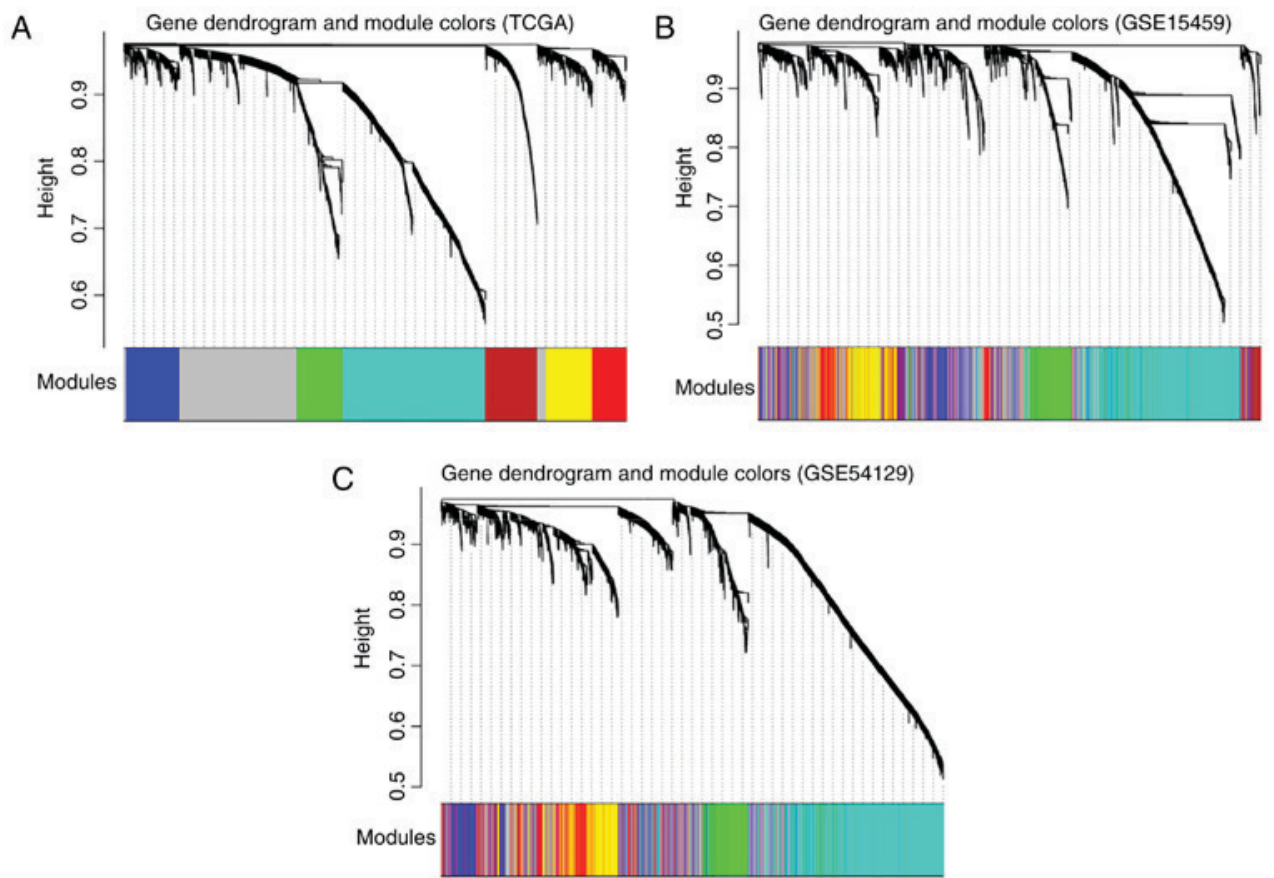

Figure 3. Module partition trees of datasets. (A) TCGA dataset, (B) GSE15459 and (C) GSE54129. Modules are indicated by different colors. TCGA, The Cancer Genome Atlas; GSE, gene set enrichment.
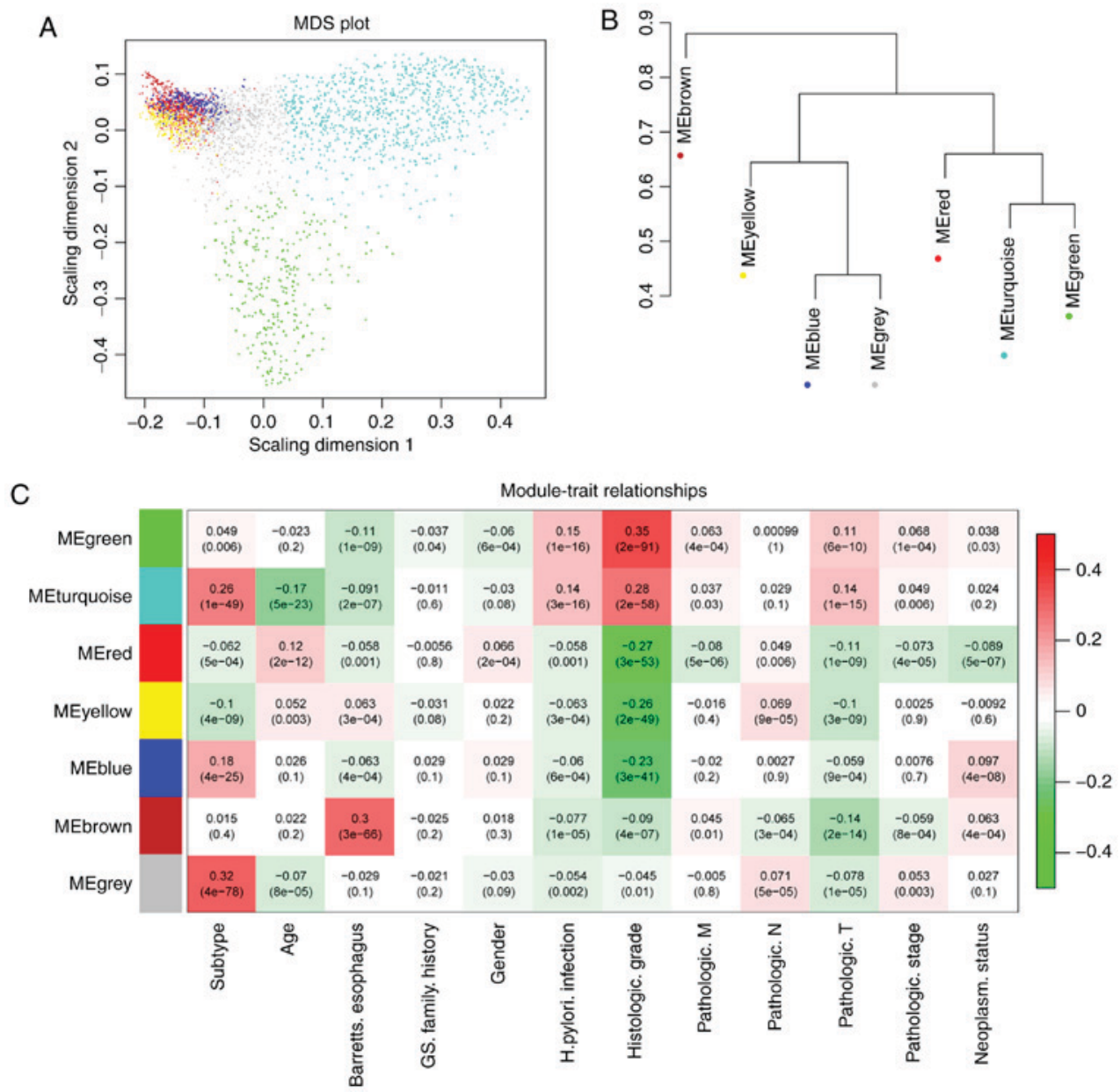

Figure 4. The association analysis of the gene modules and clinical features. (A) Multidimensional extension plot of the RNAs in each module. The horizontal and vertical axes separately represent the first and second principal components. (B) Module dendrogram of the seven modules. (C) Correlation heat map between each module and clinical factors; the horizontal and vertical axes separately represent clinical factors and modules; the change of color from green to red indicates the change of correlation from negative to positive; the numbers in grids represent correlation coefficients, and the numbers in parentheses represent significant P-values. ME, module eigengene; MDS, multidimensional scaling; TNM, Tumor Node Metastasis. 
Table I. Differentially expressed lncRNAs between the tumor and control groups in 7 modules.

\begin{tabular}{|c|c|c|c|c|c|c|c|c|c|}
\hline \multicolumn{5}{|l|}{ ad } & \\
\hline \multirow{2}{*}{ Group } & \multirow{2}{*}{ Module } & \multirow{2}{*}{ P-value } & \multirow{2}{*}{ FDR } & \multirow{2}{*}{$\log \mathrm{FC}$} & & & & & \\
\hline & & & & & CSNK1G2-AS1 & Turquoise & $3.76 \times 10^{-9}$ & $2.10 \times 10^{-8}$ & 1.2401 \\
\hline HOTAIR & Blue & $1.01 \times 10^{-55}$ & $1.46 \times 10^{-53}$ & 5.2805 & $C Y P 1 B 1-A S 1$ & Turquoise & $2.36 \times 10^{-3}$ & $4.49 \times 10^{-3}$ & -0.7444 \\
\hline$M C F 2 L-A S 1$ & Blue & $2.58 \times 10^{-15}$ & $3.74 \times 10^{-14}$ & 0.9188 & DSCR10 & Turquoise & $5.40 \times 10^{-4}$ & $1.22 \times 10^{-3}$ & 3.0388 \\
\hline GAS5 & Brown & $8.85 \times 10^{-7}$ & $3.38 \times 10^{-6}$ & 0.1977 & ENO1-AS1 & Turquoise & $1.73 \times 10^{-4}$ & $4.56 \times 10^{-4}$ & 0.4499 \\
\hline$C A S C 2$ & Green & $5.41 \times 10^{-11}$ & $3.92 \times 10^{-10}$ & 0.7532 & FBXL19-AS1 & Turquoise & $3.49 \times 10^{-32}$ & $1.01 \times 10^{-30}$ & 0.9572 \\
\hline CECR3 & Green & $2.06 \times 10^{-11}$ & $1.66 \times 10^{-10}$ & 2.8280 & $J P X$ & Turquoise & $3.27 \times 10^{-5}$ & $9.30 \times 10^{-5}$ & 0.2466 \\
\hline CPS1-IT1 & Green & $1.58 \times 10^{-2}$ & $2.48 \times 10^{-2}$ & 1.2022 & LGALS8-AS1 & Turquoise & $1.04 \times 10^{-6}$ & $3.87 \times 10^{-6}$ & 0.4645 \\
\hline$H C G 27$ & Green & $2.35 \times 10^{-3}$ & $4.49 \times 10^{-3}$ & 0.3698 & LINC00052 & Turquoise & $2.46 \times 10^{-6}$ & $8.70 \times 10^{-6}$ & 3.7146 \\
\hline$I G F 2-A S$ & Green & $3.12 \times 10^{-6}$ & $1.08 \times 10^{-5}$ & 2.2240 & LINC00161 & Turquoise & $4.63 \times 10^{-4}$ & $1.08 \times 10^{-3}$ & 0.9597 \\
\hline$I N H B A-A S 1$ & Green & $1.85 \times 10^{-2}$ & $2.86 \times 10^{-2}$ & & LINC00189 & Turquoise & $2.11 \times 10^{-8}$ & $1.09 \times 10^{-7}$ & 1.2475 \\
\hline$J A Z F 1-A S 1$ & Green & $6.36 \times 10^{-3}$ & $1.12 \times 10^{-2}$ & U & LINC00290 & Turquoise & $2.47 \times 10^{-2}$ & $3.73 \times 10^{-2}$ & 2.6024 \\
\hline & & & & -0 . & LINC00308 & Turquoise & $4.33 \times 10^{-3}$ & $7.85 \times 10^{-3}$ & 2.1851 \\
\hline$K C$ & Green & 3 & 2. & 1.8214 & LINC00309 & Turquoise & $2.65 \times 10^{-4}$ & $6.75 \times 10^{-4}$ & 2.2938 \\
\hline LINC00032 & Green & $3.31 \times 10^{-3}$ & $6.15 \times 10^{-3}$ & 0.8562 & LINC00311 & Turquoise & $1.32 \times 10^{-2}$ & $2.14 \times 10^{-2}$ & 0.6300 \\
\hline LINC00112 & Green & $1.50 \times 10^{-2}$ & $2.42 \times 10^{-2}$ & 2.1061 & LINC00323 & Turquoise & $6.57 \times 10^{-3}$ & $1.15 \times 10^{-2}$ & 0.5396 \\
\hline LINC00115 & Green & $1.66 \times 10^{-10}$ & $1.09 \times 10^{-9}$ & 0.7638 & LINC00347 & Turquoise & $1.63 \times 10^{-3}$ & $3.32 \times 10^{-3}$ & 2.4716 \\
\hline LINC00163 & Green & $2.81 \times 10^{-2}$ & $4.16 \times 10^{-2}$ & -1.2973 & LINC00471 & Turquoise & $3.59 \times 10^{-6}$ & $1.18 \times 10^{-5}$ & 1.0278 \\
\hline LINC00167 & Green & $7.42 \times 10^{-4}$ & $1.58 \times 10^{-3}$ & 0.5133 & LINC00477 & Turquoise & $4.83 \times 10^{-3}$ & $8.64 \times 10^{-3}$ & 1.4857 \\
\hline LINC00242 & Green & $1.84 \times 10^{-6}$ & $6.67 \times 10^{-6}$ & 0.6465 & LINC00479 & Turquoise & $4.59 \times 10^{-4}$ & $1.08 \times 10^{-3}$ & 1.1469 \\
\hline LINC00299 & Green & $2.20 \times 10^{-16}$ & $3.99 \times 10^{-15}$ & 1.8836 & LINC00482 & Turquoise & $1.02 \times 10^{-4}$ & $2.80 \times 10^{-4}$ & 0.8407 \\
\hline LINC00326 & Green & $1.72 \times 10^{-3}$ & $3.47 \times 10^{-3}$ & 4.7055 & LINCO0518 & Turquoise & $6.17 \times 10^{-8}$ & $2.98 \times 10^{-7}$ & 2.5336 \\
\hline LINC00330 & Green & $1.28 \times 10^{-2}$ & $2.12 \times 10^{-2}$ & -1.9390 & LINC00582 & Turquoise & $4.52 \times 10^{-4}$ & $1.08 \times 10^{-3}$ & -1.5306 \\
\hline LINC00410 & Green & $4.51 \times 10^{-7}$ & $1.92 \times 10^{-6}$ & 4.2579 & $N B R 2$ & Turquoise & $1.03 \times 10^{-9}$ & $6.49 \times 10^{-9}$ & 0.3945 \\
\hline LINC00485 & Gre & & & 4.2519 & NEAT1 & Turquoise & $1.19 \times 10^{-5}$ & $3.45 \times 10^{-5}$ & 0.2762 \\
\hline & & & & $2.05 / 8$ & NPSR 1-AS1 & Turquoise & $9.94 \times 10^{-31}$ & $2.40 \times 10^{-29}$ & 5.6701 \\
\hline LINCOO & Green & $3.22 \times 10^{-4}$ & $8.06 \times 10^{-4}$ & 1.6764 & $P C B P 1-A S 1$ & Turquoise & $3.37 \times 10^{-8}$ & $1.69 \times 10^{-7}$ & -0.4557 \\
\hline LINC00523 & Green & $1.59 \times 10^{-3}$ & $3.29 \times 10^{-3}$ & 3.1421 & RUSC1-AS1 & Turquoise & $7.90 \times 10^{-7}$ & $3.10 \times 10^{-6}$ & 0.1934 \\
\hline LINC00607 & Green & $5.97 \times 10^{-7}$ & $2.40 \times 10^{-6}$ & 1.4066 & $S T 7-A S 2$ & Turquoise & $5.65 \times 10^{-6}$ & $1.77 \times 10^{-5}$ & 0.2453 \\
\hline$W D F Y 3-A S 2$ & Green & $6.90 \times 10^{-4}$ & $1.54 \times 10^{-3}$ & -0.4393 & ZNF295-AS1 & Turquoise & $1.89 \times 10^{-3}$ & $3.74 \times 10^{-3}$ & 1.0881 \\
\hline$A D A R B 2-A S 1$ & Grey & $1.52 \times 10^{-4}$ & $4.09 \times 10^{-4}$ & 2.0305 & ZNF503-AS2 & Turquoise & $2.98 \times 10^{-2}$ & $4.35 \times 10^{-2}$ & -0.1638 \\
\hline TP53TG1 & Grey & $5.11 \times 10^{-6}$ & $1.65 \times 10^{-5}$ & -0.2026 & C20orf203 & Yellow & $3.31 \times 10^{-2}$ & $4.75 \times 10^{-2}$ & 0.7754 \\
\hline TTTY14 & Grey & $4.86 \times 10^{-4}$ & $1.12 \times 10^{-3}$ & -1.0856 & DLEU2 & Yellow & $1.01 \times 10^{-35}$ & $4.88 \times 10^{-34}$ & 1.0471 \\
\hline AGPAT4-IT1 & Red & $5.75 \times 10^{-6}$ & $1.77 \times 10^{-5}$ & 0.8612 & FAM201A & Yellow & $1.42 \times 10^{-9}$ & $8.58 \times 10^{-9}$ & 0.8587 \\
\hline BPESC1 & Red & $1.12 \times 10^{-3}$ & $2.35 \times 10^{-3}$ & 1.7243 & FАМб6C & Yellow & $1.54 \times 10^{-2}$ & $2.45 \times 10^{-2}$ & -0.5395 \\
\hline$B V E S-A S 1$ & Red & $1.82 \times 10^{-4}$ & $4.71 \times 10^{-4}$ & -1.6299 & $H C G 4 B$ & Yellow & $2.42 \times 10^{-7}$ & $1.10 \times 10^{-6}$ & 1.1311 \\
\hline DGCR 5 & Red & $6.81 \times 10^{-15}$ & $8.98 \times 10^{-14}$ & $\begin{array}{r}-1.0299 \\
17023\end{array}$ & HCG9 & Yellow & $7.28 \times 10^{-3}$ & $1.24 \times 10^{-2}$ & 0.5412 \\
\hline$D S C R 9$ & Red & $94 \times 10^{-14}$ & $1.08 \times 10^{-12}$ & 5 & HCP5 & Yellow & $3.42 \times 10^{-6}$ & $1.15 \times 10^{-5}$ & 0.4477 \\
\hline & & $10^{-2}$ & $5.00 \times 10$ & & INEI & Yellow & $4.51 \times 10^{-12}$ & $3.85 \times 10^{-11}$ & 0.8886 \\
\hline EFB41L & Red & & & -0.0787 & KIF 25-AS1 & Yellow & $1.51 \times 10^{-7}$ & $7.06 \times 10^{-7}$ & 1.7721 \\
\hline HCG18 & Red & $1.24 \times 10^{-12}$ & $1.20 \times 10^{-11}$ & 0.4696 & LINC00174 & Yellow & $2.83 \times 10^{-13}$ & $2.93 \times 10^{-12}$ & 0.8178 \\
\hline LINC00029 & Red & $2.01 \times 10^{-3}$ & $3.94 \times 10^{-3}$ & 1.7851 & LINC00265 & Yellow & $4.80 \times 10^{-7}$ & $1.99 \times 10^{-6}$ & 0.5363 \\
\hline LINC00467 & Red & $1.06 \times 10^{-10}$ & $7.32 \times 10^{-10}$ & 0.4418 & LINC00599 & Yellow & $9.87 \times 10^{-3}$ & $1.66 \times 10^{-2}$ & 1.5708 \\
\hline LINC00470 & Red & $5.90 \times 10^{-6}$ & $1.78 \times 10^{-5}$ & 1.6068 & LINC00606 & Yellow & $1.28 \times 10^{-2}$ & $2.12 \times 10^{-2}$ & 4.1423 \\
\hline LINC00487 & Red & $2.29 \times 10^{-2}$ & $3.49 \times 10^{-2}$ & 0.9837 & LY86-AS1 & Yellow & $7.20 \times 10^{-4}$ & $1.56 \times 10^{-3}$ & 1.2773 \\
\hline LINC00574 & Red & $8.80 \times 10^{-5}$ & $2.45 \times 10^{-4}$ & 0.7952 & PART1 & Yellow & $6.79 \times 10^{-6}$ & $2.01 \times 10^{-5}$ & -1.5909 \\
\hline$M A G I 2-A S 3$ & Red & $3.00 \times 10^{-2}$ & $4.35 \times 10^{-2}$ & -0.4123 & RHPN1-AS1 & Yellow & $6.31 \times 10^{-33}$ & $2.29 \times 10^{-31}$ & 1.5361 \\
\hline MIR17HG & Red & $2.02 \times 10^{-27}$ & $4.18 \times 10^{-26}$ & 1.5489 & SND1-IT1 & Yellow & $1.31 \times 10^{-13}$ & $1.46 \times 10^{-12}$ & 1.1209 \\
\hline MORC2-ASI & Red & $3.08 \times 10^{-16}$ & $4.96 \times 10^{-15}$ & & SOX2-OT & Yellow & $2.75 \times 10^{-2}$ & $4.11 \times 10^{-2}$ & -0.4175 \\
\hline$S H A N K 2-A S 3$ & Red & $1.49 \times 10^{-8}$ & $8.00 \times 10^{-8}$ & & TP73-AS1 & Yellow & $4.11 \times 10^{-3}$ & $7.55 \times 10^{-3}$ & -0.3515 \\
\hline & & & & 34 & TUG1 & Yellow & $3.08 \times 10^{-11}$ & $2.35 \times 10^{-10}$ & 0.2871 \\
\hline & $\mathrm{F}$ & & & & $Z N F 252 P-A S 1$ & Yellow & $4.75 \times 10^{-37}$ & $3.44 \times 10^{-35}$ & 1.4731 \\
\hline & O1se & 1 & 4 & & & & & & \\
\hline C20orf10 & loise & & & -1.8050 & & & & & ES \\
\hline$C C D C 26$ & Turquoise & $6.66 \times 10^{-3}$ & $1.15 \times 10^{-2}$ & 0.9937 & & & & & roject. \\
\hline$C I R B P-A S 1$ & Turquoise & $2.95 \times 10^{-9}$ & $1.71 \times 10^{-8}$ & 0.6040 & & & & & 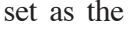 \\
\hline$C R N D E$ & Turquoise & $2.43 \times 10^{-3}$ & $4.58 \times 10^{-3}$ & 0.4485 & & & & & \\
\hline
\end{tabular}

Table I. Continued.

Differentially expressed lncRNAs were screened using the MetaDE.ES algorithm in the R package MetaDE (version 1.0.5; https://cran.r-project. org/web/packages/MetaDE/), $\mathrm{P}<0.05$ and $\mathrm{FDR}<0.05$ were set as the 

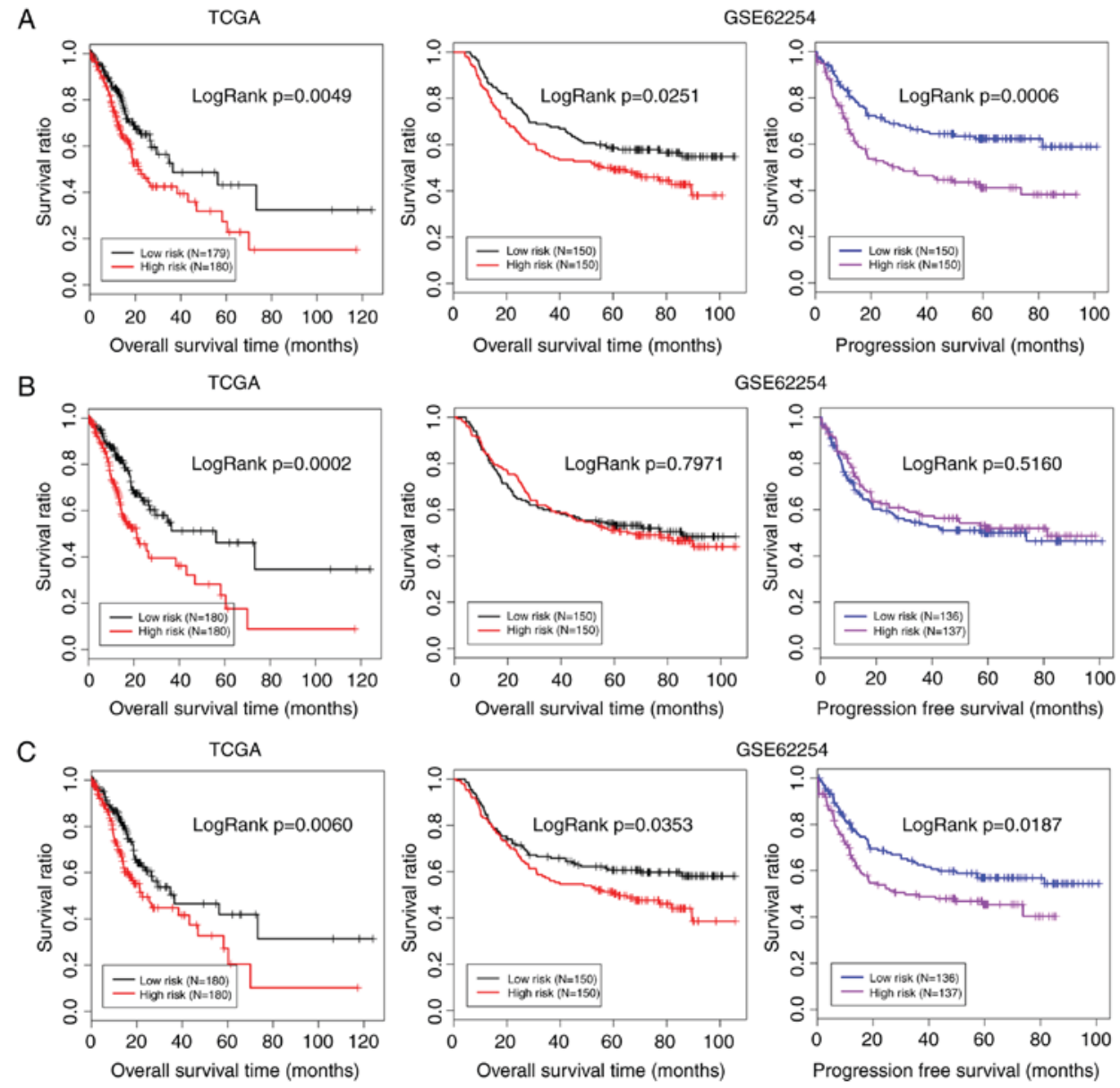

Figure 5. Kaplan-Meier survival curves of the correlations between patient survival and the risk grouping based on the risk score systems of the modules. (A) green, (B) turquoise and (C) green + turquoise modules. Left, middle and right curves represent the overall survival time of TCGA dataset, the overall survival time of GSE62254 and the progression-free survival time of GSE62254, respectively. The black and blue lines represent the low-risk group, and the red and purple lines represent the high-risk group. TCGA, The Cancer Genome Atlas; GSE, gene set enrichment.

dataset using univariate Cox regression analysis. Among the 12 prognosis-associated lncRNAs, 5 belonged to the green module and 7 were from the turquoise module.

Construction and assessment of risk score system. Based on the expression levels of 12 prognosis-associated lncRNAs in TCGA dataset, the optimal lncRNA combinations that correlated with prognosis were selected using the Cox-PH model. 5-lncRNA, 5-lncRNA and 8-1ncRNA (Table III) optimal combinations were separately screened from the prognosis-associated lncRNAs in the green, turquoise and green + turquoise modules, respectively. The risk score systems based on each optimal lncRNA combination were as follows: Risk score (green module $)=(-0.9059377) \times \operatorname{Exp}_{\text {ITPK } 1-\mathrm{AS} 1}$ $+(3.3537827) \operatorname{Exp}_{\text {KCNQ1DN }}+(-2.1388024) \times \operatorname{Exp}_{\text {LINC00167 }}$ $+(-1.037547) \times \operatorname{Exp}_{\text {LINC00173 }}+(1.9587271) \times \operatorname{Exp}_{\text {LINC00307 }}$ Risk score (turquoise module $)=(-0.268429) \times \operatorname{Exp}_{\text {ASMTL-AS1 }}$ $+(-0.3410407) \times \operatorname{Exp}_{\mathrm{CIRBP}-\mathrm{AS} 1}+(1.0926567) \times \operatorname{Exp}_{\mathrm{DSCR} 10}$ $+(-0.3433227) \times \operatorname{Exp}_{\text {JPX }}+(0.5437058) \times \operatorname{Exp}_{\text {LINC00479}}$. Risk score $($ green + turquoise module $)=(1.9685961) \times \operatorname{Exp}_{\text {KCNQ1DN }}$ $+(-0.6567239) \times \operatorname{Exp}_{\text {LINC00167 }}+(-0.4293328) \times \operatorname{Exp}_{\text {LINC00173 }}+$ $(-0.246053) \times \operatorname{Exp}_{\text {ASMTL-AS } 1}+(-0.25746771) \times \operatorname{Exp}_{\text {CIRBP-AS } 1}+$ $(0.7023183) \times \operatorname{Exp}_{\mathrm{DSCR} 10}+(-0.3204003) \times \operatorname{Exp}_{\mathrm{JPX}}+(0.5495452)$ $\mathrm{x} \operatorname{Exp}_{\text {LINC00479. }}$
Based on the three risk score systems, the risk scores of the samples in TCGA dataset were calculated. The samples in the TCGA dataset were divided into high-risk and low-risk groups according to the median of their risk scores. Kaplan-Meier survival curves were used to evaluate the correlation between the overall survival of the samples and the two groups. The results revealed that the risk score system based on the optimal lncRNA combination [including ITPK1 antisense RNA 1 (ITPK1-AS1), KCNQ1 downstream neighbor $(K C N Q 1 D N)$, long intergenic non-protein coding RNA 167 (LINC00167), LINC00173 and LINC00307] of the green module had the most significant predictive effect; therefore, the risk score system of the green module was the optimal system (Fig. 5). In this risk score system, the low-risk group (mean overall survival time, 16.71 18.26 months) had a greater overall survival time compared with the high-risk group (mean overall survival time, 13.63 \pm 15.76 months) for the TCGA training dataset. In addition, the correlation between overall survival and the two groups was significant $(\mathrm{P}=0.0049)$. For the validation dataset GSE62254, the low-risk group (mean overall survival time, $57.13 \pm 30.88$ months; mean progression-free survival time, $42.34 \pm 30.26$ months) also had a greater overall survival time and progression-free survival time relative to the high-risk group (mean overall survival 
Table II. Stabilities and functional annotations of the 7 modules of TCGA dataset.

\begin{tabular}{|c|c|c|c|c|c|c|}
\hline Module & Color & $\begin{array}{l}\text { Module } \\
\text { size, } \mathrm{n}\end{array}$ & mRNA & lncRNA & $\begin{array}{c}\text { Preservation } \\
\text { Z-score }\end{array}$ & Module annotation \\
\hline 1 & Blue & 336 & 334 & 2 & 9.7094 & Pattern specification process \\
\hline 2 & Brown & 331 & 328 & 3 & 1.2017 & Epithelium development \\
\hline 3 & Green & 318 & 288 & 30 & 19.0215 & Immune response \\
\hline 4 & Grey & 2,856 & 2,822 & 34 & 4.2851 & Cell-cell signaling \\
\hline 5 & Red & 250 & 213 & 37 & 13.2273 & Digestive system process \\
\hline 6 & Turquoise & 956 & 910 & 46 & 27.4163 & Cell adhesion \\
\hline 7 & Yellow & 326 & 294 & 32 & 15.7692 & Digestion \\
\hline
\end{tabular}

$\mathrm{Z}<5$ indicates that the module is unstable; $\mathrm{Z}>5$ indicates that the module is stable, and $\mathrm{Z}>10$ indicates that the module is highly stable. Module annotation indicates the functional terms enriched for the modules. TCGA, The Cancer Genome Atlas; lncRNA, long non-coding RNA.

Table III. Optimal lncRNAs screened from the prognosis-associated lncRNAs in green, turquoise and green + turquoise modules

\begin{tabular}{|c|c|c|c|c|}
\hline Modules & lncRNA & $\beta$-value & P-values & Hazard ratio $(95 \% \mathrm{CI})$ \\
\hline \multirow[t]{5}{*}{ Green } & ITPK1-AS1 & -0.9059 & 0.0496 & $0.0777(0.0039-1.5300)$ \\
\hline & $K C N Q 1 D N$ & 3.3538 & 0.0050 & $13.7200(2.1360-18.1400)$ \\
\hline & LINC00167 & -2.1388 & 0.0284 & $0.0500(0.0035-0.7191)$ \\
\hline & LINC00173 & -1.0376 & 0.0480 & $0.4930(0.2229-1.0900)$ \\
\hline & LINC00307 & 1.9587 & 0.0357 & $2.0260(1.0430-3.9380)$ \\
\hline \multirow[t]{5}{*}{ Turquoise } & $A S M T L-A S 1$ & -0.2684 & 0.0270 & $0.6392(0.4302-0.9498)$ \\
\hline & $C I R B P-A S 1$ & -0.3410 & 0.0458 & $0.6489(0.4146-1.0160)$ \\
\hline & DSCR10 & 1.0927 & 0.0039 & $4.4030(1.5400-12.5900)$ \\
\hline & $J P X$ & -0.3433 & 0.0470 & $0.6624(0.4243 \sim 1.0340)$ \\
\hline & LINC00479 & 0.5437 & 0.0231 & $1.9880(1.0900-3.6260)$ \\
\hline \multirow[t]{8}{*}{ Green + turquoise } & $K C N Q 1 D N$ & 1.9686 & 0.0029 & $3.3500(1.3101-5.3910)$ \\
\hline & LINC00167 & -0.6567 & 0.0476 & $0.0790(0.0048-1.3100)$ \\
\hline & LINC00173 & -0.4293 & 0.0482 & $0.4338(0.1693-1.1110)$ \\
\hline & $A S M T L-A S 1$ & -0.2461 & 0.0238 & $0.7758(0.5088-1.1830)$ \\
\hline & $C I R B P-A S 1$ & -0.2575 & 0.0489 & $0.8482(0.5322-1.3520)$ \\
\hline & DSCR10 & 0.7023 & 0.0208 & $2.2280(1.6399-7.7580)$ \\
\hline & $J P X$ & -0.3204 & 0.0131 & $0.6847(0.4187-1.1200)$ \\
\hline & LINC00479 & 0.5495 & 0.0337 & $1.9057(1.0510-3.4550)$ \\
\hline
\end{tabular}

P-values were generated by univariate cox regression, with $\mathrm{P}<0.05$ as the threshold. lncRNA, long non-coding RNA; CI, confidence interval.

time, 46.98 \pm 29.97 months; mean progression-free survival time, 30.91 \pm 27.97 months). Similarly, the two groups were significantly correlated with overall survival time $(\mathrm{P}=0.0251)$ and progression-free survival time $(\mathrm{P}=0.0006)$. Additionally, the associations between the risk score and survival status/lncRNA expression are displayed in Fig. 6. The risk score altered from low to high on the vertical axis; in the middle panels, red represents mortality, and black represents survival, which represented the distribution of mortality and survival at high and low risk in addition to the distribution of survival time. Fig. 6 reveals the expression trend of 5 genes from low risk to high risk (for example, LINC00307 expression tends to be decreased, while $K C N Q 1 D N$ expression tends to be increased).
Differential expression analysis. There were a total of 1,105 consensus DE-RNAs (all were mRNAs) in the GSE65801, GSE29998, GSE33335 and GSE27342 datasets, including 22 mRNAs (2 upregulated and 20 downregulated) in the green module. The clustering heat map demonstrates that the different degrees and dysregulation directions of the DE-RNAs were essentially the same in the 4 datasets (Fig. 7).

Analysis of IncRNA-associated pathways. Based on the correlation coefficients of the 5 optimal lncRNAs in the green module, and the $22 \mathrm{mRNAs}$ obtained as aforementioned, the lncRNA-mRNA network (involving 106 nodes) was constructed (Fig. 8A). GSEA analysis illustrated that 4 pathways ['cell adhesion molecules (CAMS)', 'cytokine-cytokine 
A1

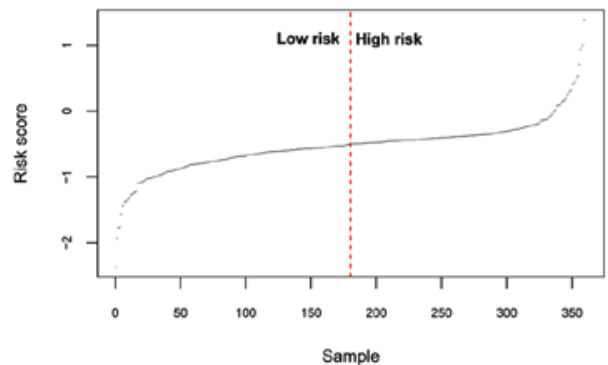

A2

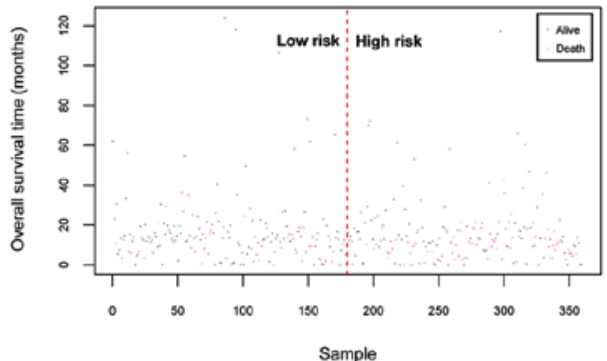

A3

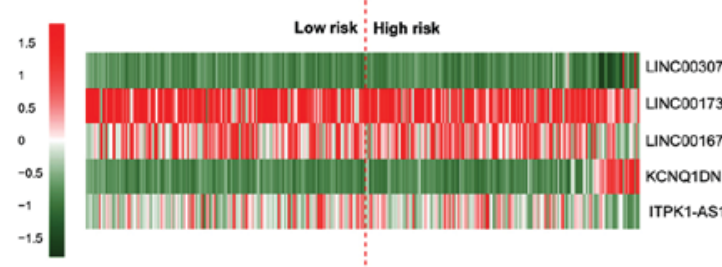

B1

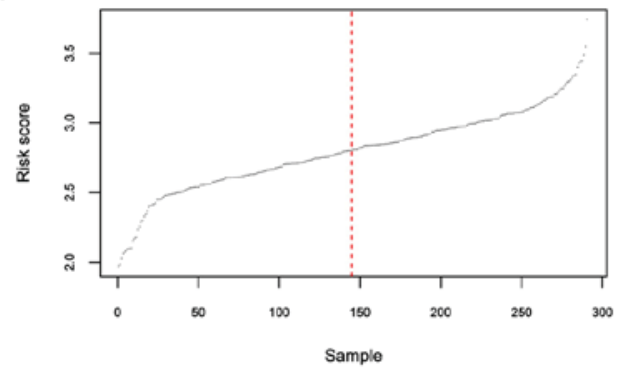

B2

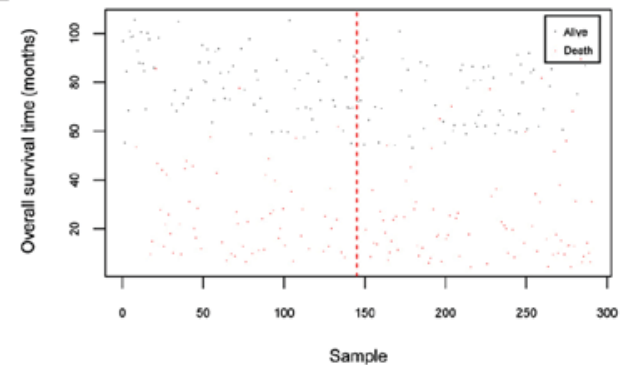

B3

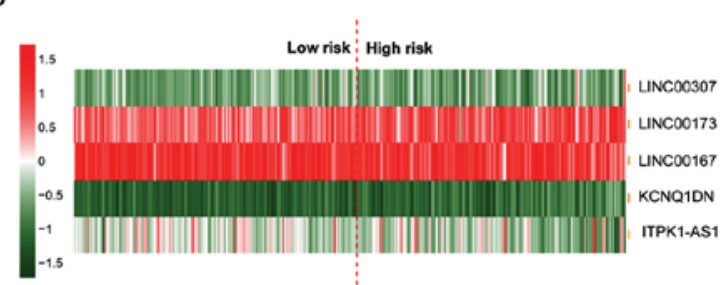

Figure 6. Associations between risk score and survival status and/or lncRNA expression in the datasets. (A) the training set and (B), the validation set. A1 and B1 represents the risk score: The horizontal axis represents the sample and the vertical axis represents the risk score. A2 and B2 represents survival status: The horizontal axis represents the sample and the vertical axis represents the survival time. A3 and B3 represents the expression levels of 5 lncRNAs: The horizontal axis represents the sample and the vertical axis represents the expression level of the gene. Red means high expression; green stands for low expression. 1ncRNA, long non-coding RNA; ITPK1-AS1, ITPK1 antisense RNA 1; KCNQ1DN, KCNQ1 downstream neighbor; LINC00167, long intergenic non-protein coding RNA 167.

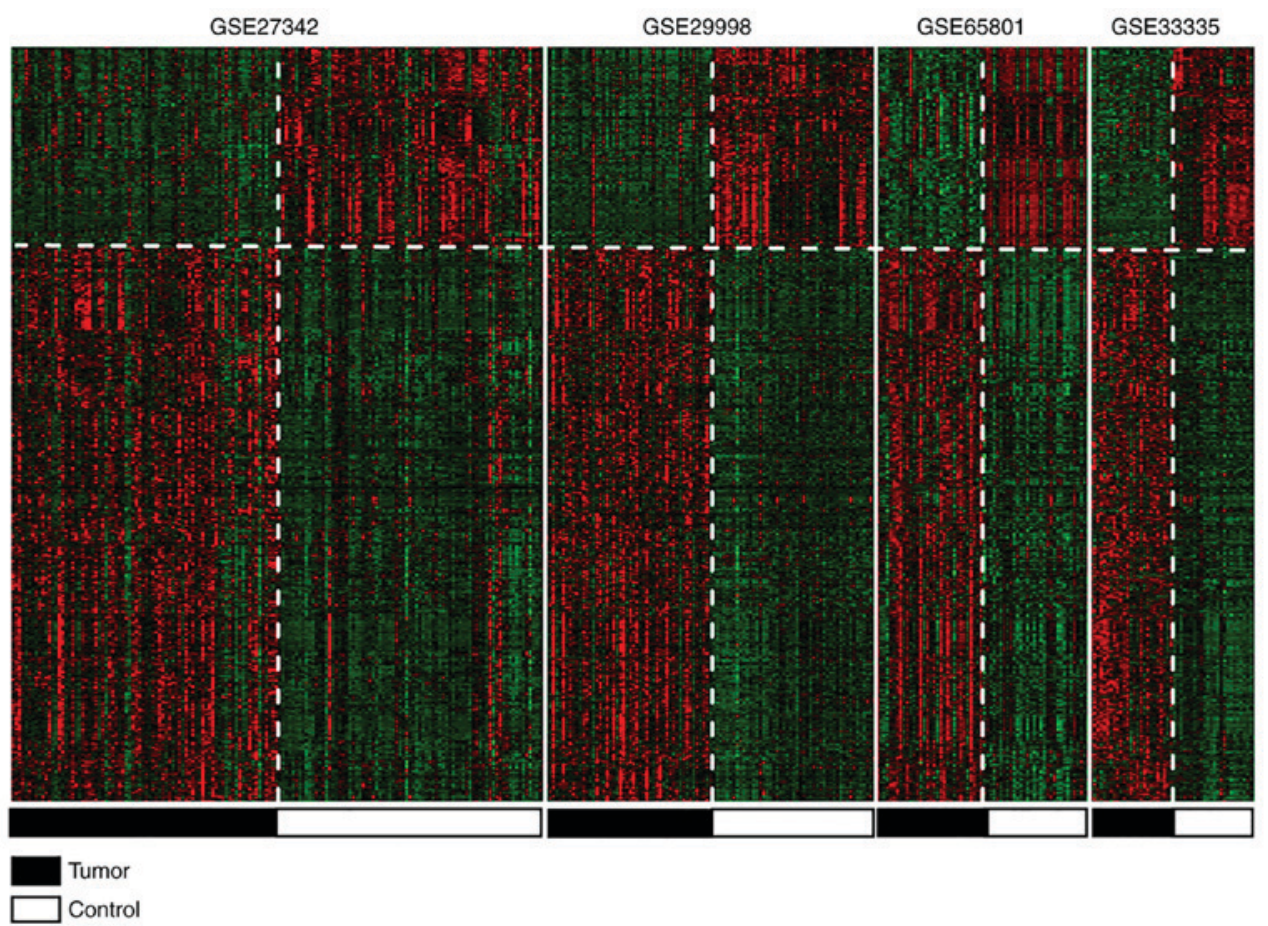

Figure 7. Clustering heat maps of the consensus differentially expressed RNAs in the GSE27342, GSE29998, GSE65801 and GSE33335 datasets. Black and white represent tumor and control samples, respectively. Red means high expression; green stands for low expression. GSE, gene set enrichment. 
Table IV. Pathways that positively correlate with LINC00167, LINC00173 and LINC00307.

\begin{tabular}{|c|c|c|c|c|c|c|c|c|c|}
\hline \multirow[b]{2}{*}{ Pathway } & \multicolumn{3}{|c|}{ LINC00167 } & \multicolumn{3}{|c|}{ LINC00173 } & \multicolumn{3}{|c|}{ LINC00307 } \\
\hline & ES & NES & P-value & ES & NES & P-value & ES & NES & P-value \\
\hline Cell adhesion molecules & 0.1598 & 1.1790 & 0.0096 & 0.1654 & 1.3800 & 0.0011 & 0.1465 & 0.8646 & 0.0058 \\
\hline Cytokine-cytokine receptor interaction & 0.2250 & 0.9947 & 0.0451 & 0.2052 & 1.0447 & 0.0357 & 0.1136 & 0.7058 & 0.0484 \\
\hline Chemokine signaling pathway & 0.2305 & 1.0060 & 0.0429 & 0.1052 & 0.5347 & 0.0469 & -0.1560 & -1.0490 & 0.0335 \\
\hline Leukocyte transendothelial migration & -0.1690 & -0.7141 & 0.0461 & 0.2331 & 0.1563 & 0.0137 & -0.1860 & -0.7750 & 0.0480 \\
\hline
\end{tabular}

P-values were generated by GSEA analysis, with $\mathrm{P}<0.05$ as the threshold. ES, enrichment score; NES, normalized enrichment score.

A

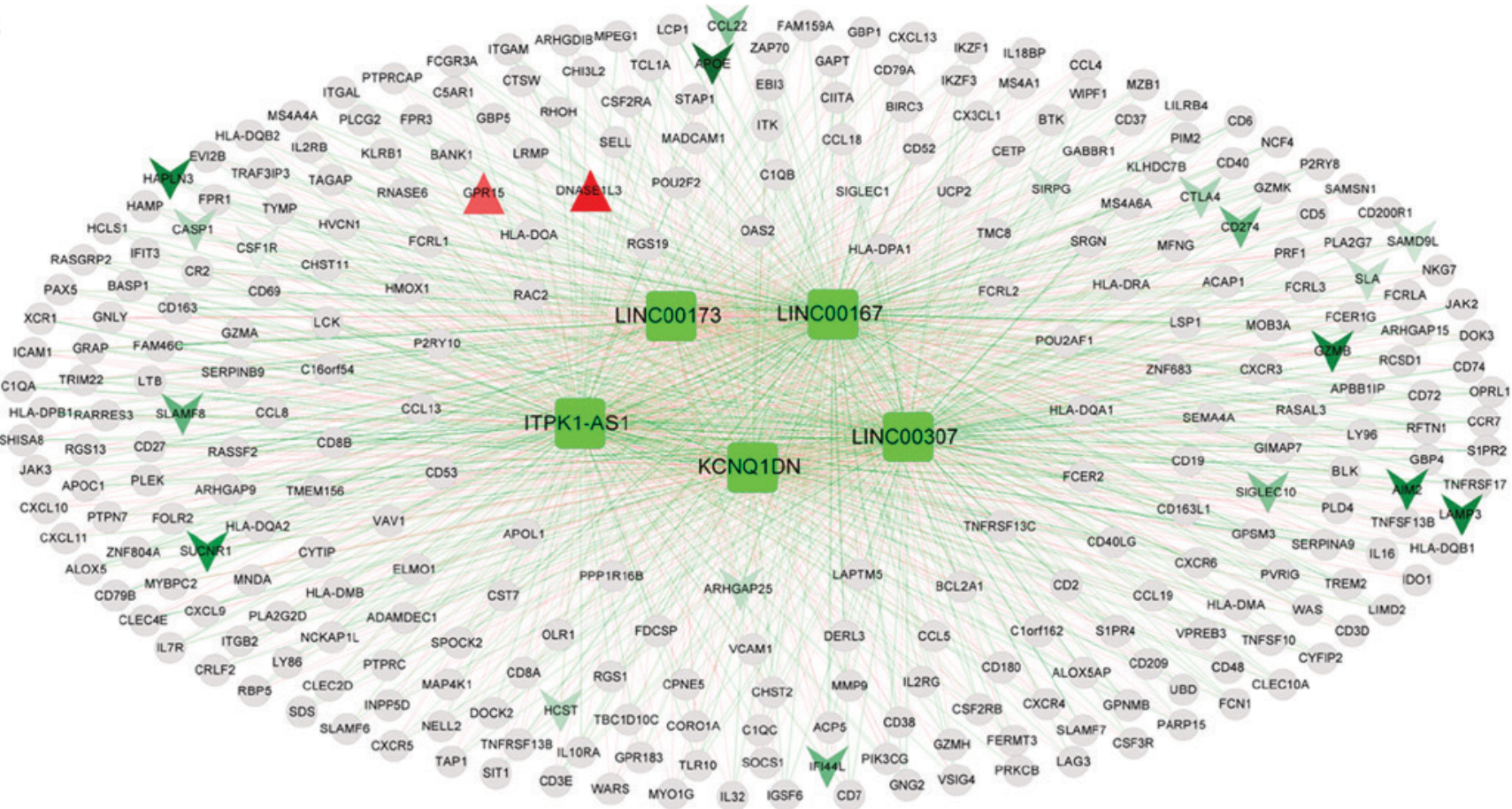

B LINC00167

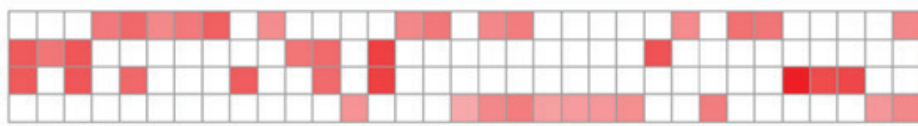

Cell adhesion molecules (CAM) Chemokineing pathway Cytokine-cytokine receptor interaction

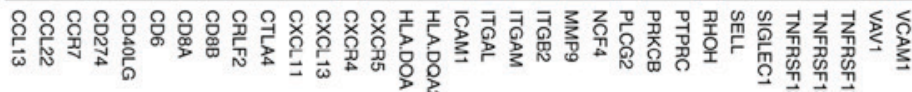
Leukocyte transendothelial migration
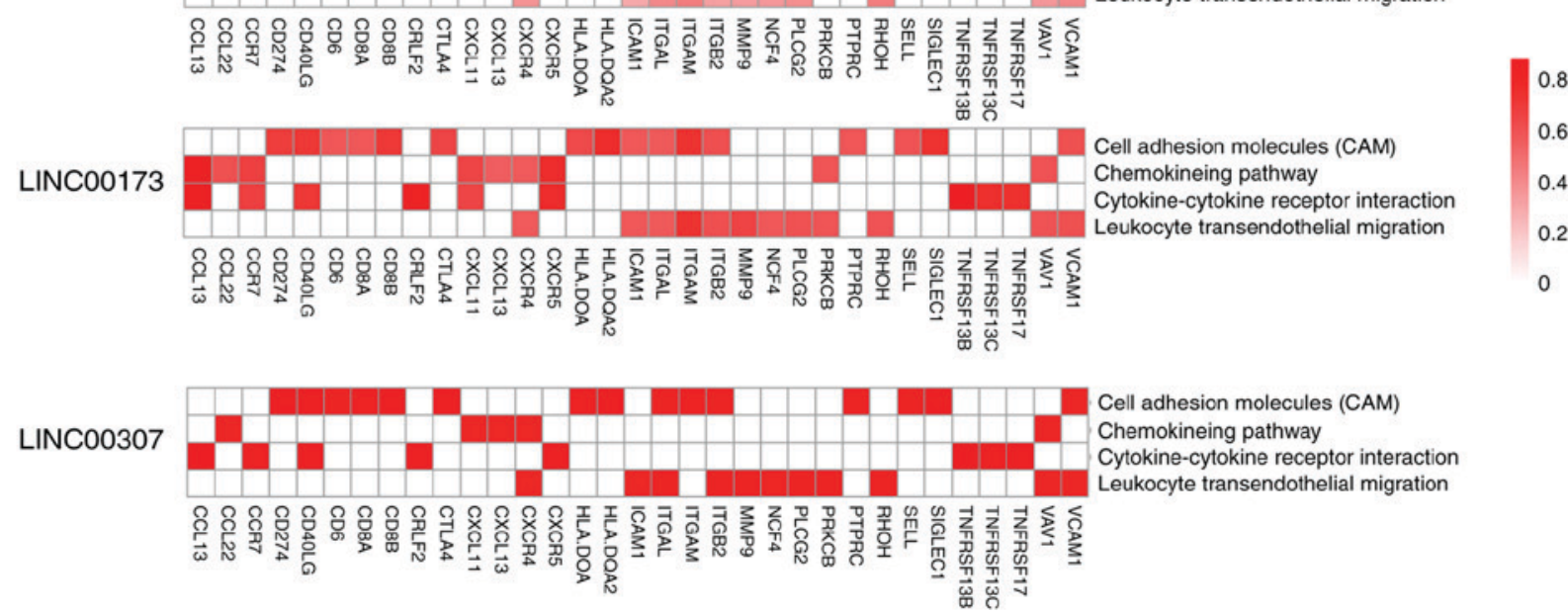

Figure 8. (A) lncRNA-mRNA network and gene heat map. The lncRNA-mRNA network is based on the green module. Grey circles represent non-consensus differentially expressed RNAs. Red regular triangles and green inverted triangles represent upregulated RNAs and downregulated RNAs, respectively. Green squares represent the 5 optimal lncRNAs in the green module. Red and green lines represent positive and negative correlations, respectively. (B) Heat map of the genes involved in the 1ncRNA-associated pathways. The deeper the red, the higher the positive correlation. lncRNA, long non-coding RNA; ITPK1-AS1, ITPK1 antisense RNA 1; KCNQ1DN, KCNQ1 downstream neighbor; LINC00167, long intergenic non-protein coding RNA 167. 
receptor interaction', the 'chemokine signaling pathway' and 'leukocyte transendothelial migration'] had significant positive associations with 3 lncRNAs (LINC00167, LINC00173 and LINC00307) (Table IV). Moreover, the 4 pathways involved a total of 32 genes [including chemokine (C-C motif) ligand 22 (CCL22), chemokine (C-C motif) receptor 7 (CCR7), cluster of differentiation (CD) 274 molecule (CD274), CD40 ligand (CD4OLG), chemokine (C-X-C motif) ligand 13, CXCL13; chemokine (C-X-C motif) receptor 5 (CXCR5), intercellular adhesion molecule 1 (ICAMI), matrix metalloproteinase 9 (MMP9) and vascular cell adhesion molecule 1 (VCAM1)], and these genes associated positively with the 4 pathways (Fig. 8B). Therefore, it was speculated that LINC00167, LINC00173 and LINC00307 may possess the same association directions with the 4 pathways and the 32 genes, and are involved in GC progression via these pathways.

\section{Discussion}

In the present study, 5 stable modules (blue, green, red, turquoise and yellow) were identified using WGCNA. In particular, the green and turquoise modules associated significantly with histological grade. Subsequently, 12 prognosis-associated lncRNAs (5 lncRNAs in the green module and seven lncRNAs in the turquoise module) were identified. Moreover, 5-lncRNA, 5-lncRNA and 8-lncRNA optimal combinations were screened separately from the prognosis-associated lncRNAs in the green, turquoise and green + turquoise modules, respectively, which were used to construct risk score systems. Notably, the risk score system based on the optimal 1ncRNA combination (including ITPK1-AS1, KCNQ1DN, LINC00167, LINC00173 and LINC00307) of the green module had the most significant predictive effect and was thus identified as the optimal system. Differential expression analysis indicated that there were 1,105 consensus DE-RNAs in the GSE65801, GSE29998, GSE33335 and GSE27342 datasets. Following the construction of the lncRNA-mRNA network, 4 pathways had significantly positive associations with LINC00167, LINC00173 and LINC00307. Moreover, the 32 genes involved in the 4 pathways associated positively with the pathways.

Potassium voltage-gated channel subfamily E regulatory subunit 2 (KCNE2) is the $\beta$ subunit of potassium voltage-gated channel subfamily Q member 1 (KCNQ1) in gastric parietal cells, and KCNQ1/KCNE2 is activated (accompanied with acid secretion) by certain pathways $(50,51)$. Through mediating the expression of $K C N Q 1$, atrial natriuretic peptide serves a role in the proliferation of the GC AGS cell line (52). KCNQ1 and insulin-like growth factor 2 mRNA-binding protein 2 polymorphisms may serve as independent predictive factors for chemotherapeutic response, and glucokinase (hexokinase 4) regulator polymorphisms may independently predict the survival of patients with metastatic GC (53). The KCNQ1 protein level was decreased in colorectal cancer samples, and was associated significantly with the unfavorable overall survival of patients with colorectal cancer (54). These observations demonstrated that $K C N Q 1 D N$ may be involved in the prognosis of GC.

CCL22 functions in the development of GC by increasing the number of regulatory T cells, and CCL22 levels in sera predict the metastasis and recurrence of GC (55). CCR7 causes epithelial-mesenchymal transition by promoting Snail expression, which results in the migration and invasion of $\mathrm{GC}$ cells $(56,57)$. A somatic mutation in $C D 274$ induces its overexpression by disturbing $m i R-570$ binding, and subsequently promotes immune evasion in GC by suppressing the activation and proliferation of $\mathrm{T}$ cells (58). The expression level of CXCL13 is a promising prognostic marker for patients with GC following surgical resection, and may be used to predict the response of these patients to postoperative adjuvant chemotherapy (59). $C D 40$ contributes to $C X C R 5$ expression, and the migration and accumulation of myeloid-derived suppressor cells in $\mathrm{GC}$, indicating that $C D 40$ may promote tumor growth by influencing immune evasion $(60,61)$. ICAM1 overexpression is induced by leptin via the Rho/Rho-associated protein kinase pathway, which contributes to tumor cell migration in patients with GC (62). MMP9 in the blood has been identified as a novel tumor marker; in particular, the plasma level of $M M P 9$ is a more effective predictor of $\mathrm{GC}$ development and progression compared with its serum level $(63,64)$. VCAMI functions in the perineural invasion (PNI) of GC by mediating the interaction between tumor cells and neural cells; therefore, VCAMI inhibition suggests a promising approach for the treatment of PNI in patients with GC (65). LINC00167, LINC00173 and LINC00307 had the same association directions with the 4 pathways and 32 genes (including CCL22, CCR7, CD274, CD40LG, CXCL13, CXCR5, ICAM1, MMP9 and VCAM1), suggesting that LINC00167, LINC00173 and LINC00307 may associate positively with GC through their participation in the 4 pathways, and by mediating the expression of these genes.

Certain limitations of the present study should be considered. Bioinformatics analyses were used to obtain these results, and no experimental research was performed. Platform differences and data heterogeneities of the datasets may have influenced the accuracy of the risk score system. Therefore, further experiments are required to confirm the results.

In conclusion, 12 prognosis-associated lncRNAs were identified from the green and turquoise modules. In addition, the optimal risk score system may be used to predict the prognosis of patients with GC. IncRNAs ITPK1-AS1, KCNQ1DN, LINC00167, LINC00173 and LINC00307 may serve important roles in the pathogenesis of GC.

\section{Acknowledgements}

Not applicable.

\section{Funding}

The present study was supported by the National Natural Science Foundation of China (grant no. 81772955), the Natural Science Foundation of Shanghai (grant no. 17ZR1439300) and the Scientific Research Program of Shanghai Municipal Commission of Health and Family Planning (grant no. 201640269).

\section{Availability of data and materials}

The datasets used during the current study are available from the corresponding author on reasonable request. 


\section{Authors' contributions}

ZH and DY performed data analyses and wrote the manuscript. YT, XZ,ZW, HF and JX contributed significantly in data analyses and manuscript revision. ZZ and QC conceived and designed the study. All authors read and approved the final manuscript.

\section{Ethics approval and consent to participate}

In the original article of the datasets, the trials were approved by the local institutional review boards of all participating centers, and informed consent was obtained from all patients.

\section{Patient consent for publication}

Not applicable.

\section{Competing interests}

The authors declare that they have no competing interests.

\section{References}

1. Van Cutsem E, Sagaert X, Topal B, Haustermans K and Prenen H: Gastric cancer. Lancet 388: 2654-2664, 2016.

2. Siegel R, Ma J, Zou Z and Jemal A: Cancer statistics, 2014. CA Cancer J Clin 64: 9-29, 2014.

3. Rugge M, Fassan M and Graham DY: Epidemiology of gastric cancer. Gastric Cancer Springer, pp23-34, 2015.

4. Feng $\mathrm{H}$, Weng $X$, Wang $Z$ and Zhang D: Relationship between living, dietary habits and gastric cancer by case-control study in residents of Zhangzhou City. Strait J Prev Med 3: 12-14, 2016 (In Chinese)

5. González CA, Sala N and Rokkas T: Gastric cancer: Epidemiologic aspects. Helicobacter 18 (Suppl 1): S34-S38, 2013

6. Orditura M, Galizia G, Sforza V, Gambardella V, Fabozzi A, Laterza MM, Andreozzi F, Ventriglia J, Savastano B, Mabilia A, et al: Treatment of gastric cancer. World J Gastroenterol 20: 1635-1649, 2014.

7. ENCODEProject Consortium, Birney E, Stamatoyannopoulos JA, Dutta A, Guigó R, Gingeras TR, Margulies EH, Weng Z, Snyder M, Dermitzakis ET, et al: Identification and analysis of functional elements in $1 \%$ of the human genome by the ENCODE pilot project. Nature 447: 799-816, 2007.

8. Johnson JM, Edwards S, Shoemaker D and Schadt EE: Dark matter in the genome: Evidence of widespread transcription detected by microarray tiling experiments. Trends Genet 21: 93-102, 2005.

9. Fatica A and Bozzoni I: Long non-coding RNAs: New players in cell differentiation and development. Nat Rev Genet 15: 7-21, 2014

10. Mattick JS, Amaral PP, Dinger ME, Mercer TR and Mehler MF: RNA regulation of epigenetic processes. Bioessays 31: 51-59, 2009.

11. Huarte M, Guttman M, Feldser D, Garber M, Koziol MJ, Kenzelmann-Broz D, Khalil AM, Zuk O, Amit I, Rabani M, et al: A large intergenic noncoding RNA induced by $\mathrm{p} 53$ mediates global gene repression in the p53 response. Cell 142: 409-419, 2010.

12. Cheetham SW, Gruhl F, Mattick JS and Dinger ME: Long noncoding RNAs and the genetics of cancer. Br J Cancer 108: $2419-2425,2013$

13. Gutschner T, Hämmerle M, Eissmann M, Hsu J, Kim Y, Hung G, Revenko A, Arun G, Stentrup M, Gross M, et al: The noncoding RNA MALAT1 is a critical regulator of the metastasis phenotype of lung cancer cells. Cancer Res 73: 1180-1189, 2013.

14. Wang Q, Zhai YY, Dai JH, Li KY, Deng Q and Han ZG: SAMD9L inactivation promotes cell proliferation via facilitating G1-S transition in hepatitis B virus-associated hepatocellular carcinoma. Int J Biol Sci 10: 807-816, 2014

15. Sun M, Xia R, Jin F, Xu T, Liu Z, De W and Liu X: Downregulated long noncoding RNA MEG3 is associated with poor prognosis and promotes cell proliferation in gastric cancer. Tumour Biol 35: 1065-1073, 2014
16. Peng W, Si S, Zhang Q, Li C, Zhao F, Wang F, Yu J and Ma R: Long non-coding RNA MEG3 functions as a competing endogenous RNA to regulate gastric cancer progression. J Exp Clin Cancer Res 34: 79, 2015.

17. Wang CY, Hua L, Yao KH, Chen JT, Zhang JJ and Hu JH: Long non-coding RNA CCAT2 is up-regulated in gastric cancer and associated with poor prognosis. Int J Clin Exp Pathol 8: 779-785, 2015.

18. Zhang EB, Kong R, Yin DD, You LH, Sun M, Han L, Xu TP, Xia R, Yang JS, De W and Chen Jf: Long noncoding RNA ANRIL indicates a poor prognosis of gastric cancer and promotes tumor growth by epigenetically silencing of miR-99a/miR-449a. Oncotarget 5: 2276-2292, 2014

19. Lan WG, Xu DH, Xu C, Ding CL, Ning FL, Zhou YL, Ma LB, Liu CM and Han X: Silencing of long non-coding RNA ANRIL inhibits the development of multidrug resistance in gastric cancer cells. Oncol Rep 36: 263-270, 2016.

20. Li L, Zhang L, Zhang Y and Zhou F: Increased expression of LncRNA BANCR is associated with clinical progression and poor prognosis in gastric cancer. Biomed Pharmacother 72: 109-112, 2015.

21. Servant N, Roméjon J, Gestraud P, La Rosa P, Lucotte G, Lair S, Bernard V, Zeitouni B, Coffin F, Jules-Clément G, et al: Bioinformatics for precision medicine in oncology: Principles and application to the SHIVA clinical trial. Front Genet 5: 152, 2014.

22. Ooi CH, Ivanova T, Wu J, Lee M, Tan IB, Tao J, Ward L, Koo JH, Gopalakrishnan V, Zhu Y, et al: Oncogenic pathway combinations predict clinical prognosis in gastric cancer. PLoS Genet 5: e1000676, 2009.

23. Tao J, Deng NT, Ramnarayanan K, Huang B, Oh HK, Leong SH, Lim SS, Tan IB, Ooi CH, Wu J, et al: CD44-SLC1A2 gene fusions in gastric cancer. Sci Transl Med 3: 77ra30, 2011.

24. Muratani M, Deng N, Ooi WF, Lin SJ, Xing M, Xu C, Qamra A, Tay ST, Malik S, Wu J, et al: Nanoscale chromatin profiling of gastric adenocarcinoma reveals cancer-associated cryptic promoters and somatically acquired regulatory elements. Nat Commun 5: 4361, 2014.

25. Chia NY, Deng N, Das K, Huang D, Hu L, Zhu Y, Lim KH, Lee MH, Wu J, Sam XX, et al: Regulatory crosstalk between lineage-survival oncogenes KLF5, GATA4 and GATA6 cooperatively promotes gastric cancer development. Gut 64: 707-719, 2015.

26. Lei Z, Tan IB, Das K, Deng N, Zouridis H, Pattison S, Chua C, Feng Z, Guan YK, Ooi CH, et al: Identification of molecular subtypes of gastric cancer with different responses to PI3-kinase inhibitors and 5-fluorouracil. Gastroenterology 145: 554-565, 2013.

27. Cristescu R, Lee J, Nebozhyn M, Kim KM, Ting JC, Wong SS, Liu J, Yue YG, Wang J, Yu K, et al: Molecular analysis of gastric cancer identifies subtypes associated with distinct clinical outcomes. Nat Med 21: 449-456, 2015.

28. Li H, Yu B, Li J, Su L, Yan M, Zhang J, Li C, Zhu Z and Liu B: Characterization of differentially expressed genes involved in pathways associated with gastric cancer. PLoS One 10: e0125013, 2015.

29. Holbrook JD, Parker JS, Gallagher KT, Halsey WS, Hughes AM, Weigman VJ, Lebowitz PF and Kumar R: Deep sequencing of gastric carcinoma reveals somatic mutations relevant to personalized medicine. J Transl Med 9: 119, 2011.

30. Cheng L, Wang P, Yang S, Yang Y, Zhang Q, Zhang W, Xiao H, Gao $\mathrm{H}$ and Zhang Q: Identification of genes with a correlation between copy number and expression in gastric cancer. BMC Med Genomics 5: 14, 2012.

31. Cheng L, Yang S, Yang Y, Zhang W, Xiao H, Gao H, Deng X and Zhang Q: Global gene expression and functional network analysis of gastric cancer identify extended pathway maps and GPRC5A as a potential biomarker. Cancer Lett 326: 105-113, 2012.

32. Cheng L, Zhang Q, Yang S, Yang Y, Zhang W, Gao H, Deng X and Zhang Q: A 4-gene panel as a marker at chromosome 8q in Asian gastric cancer patients. Genomics 102: 323-330, 2013.

33. Cui J, Chen Y, Chou WC, Sun L, Chen L, Suo J, Ni Z, Zhang M, Kong X, Hoffman LL, et al: An integrated transcriptomic and computational analysis for biomarker identification in gastric cancer. Nucleic Acids Res 39: 1197-1207, 2011.

34. Cui J,Li F, Wang G, Fang X, Puett JD and Xu Y: Gene-expression signatures can distinguish gastric cancer grades and stages. PLoS One 6: e17819, 2011. 
35. Bolstad BM, Irizarry RA, Astrand M and Speed TP: A comparison of normalization methods for high density oligonucleotide array data based on variance and bias. Bioinformatics 19 185-193, 2003

36. Irizarry RA, Bolstad BM, Collin F, Cope LM, Hobbs B and Speed TP: Summaries of Affymetrix GeneChip probe level data. Nucleic Acids Res 31: e15, 2003.

37. Ritchie ME, Phipson B, Wu D, Hu Y, Law CW, Shi W and Smyth GK: limma powers differential expression analyses for RNA-sequencing and microarray studies. Nucleic Acids Res 43: e47, 2015.

38. Larkin MA, Blackshields G, Brown NP, Chenna R, McGettigan PA, McWilliam H, Valentin F, Wallace IM, Wilm A, Lopez R, et al: Clustal W and Clustal X version 2.0. Bioinformatics 23: 2947-2948, 2007.

39. Zhou M, Guo M, He D, Wang X, Cui Y, Yang H, Hao D and Sun J: A potential signature of eight long non-coding RNAs predicts survival in patients with non-small cell lung cancer. J Transl Med 13: 231, 2015.

40. Zhou M, Xu W, Yue X, Zhao H, Wang Z, Shi H, Cheng L and Sun J: Relapse-related long non-coding RNA signature to improve prognosis prediction of lung adenocarcinoma. Oncotarget 7: 29720-29738, 2016

41. Sun C, Jiang H, Sun Z, Gui Y and Xia H: Identification of long non-coding RNAs biomarkers for early diagnosis of myocardial infarction from the dysregulated coding-non-coding co-expression network. Oncotarget 7: 73541-73551, 2016.

42. Zhai X, Xue Q, Liu Q, Guo Y and Chen Z: Colon cancer recurrence-associated genes revealed by WGCNA co-expression network analysis. Mol Med Rep 16: 6499-6505, 2017.

43. Langfelder P and Horvath S: WGCNA: An R package for weighted correlation network analysis. Bmc Bioinformatics 9: $559,2008$.

44. Wang P, Wang Y, Hang B, Zou X and Mao JH: A novel gene expression-based prognostic scoring system to predict survival in gastric cancer. Oncotarget 7: 55343-55351, 2016.

45. Goeman JJ: L1 penalized estimation in the Cox proportional hazards model. Biom J 52: 70-84, 2010.

46. Knafl GJ, Dixon JK, O'Malley JP, Grey M, Deatrick JA Gallo A and Knafl KA: Scale development based on likelihood cross-validation. Stat Methods Med Res 21: 599-619, 2012.

47. Qi C, Hong L, Cheng Z and Yin Q: Identification of metastasis-associated genes in colorectal cancer using metaDE and survival analysis. Oncol Lett 11: 568-574, 2016.

48. Huang J, Deng Q, Wang Q, Li KY, Dai JH, Li N, Zhu ZD, Zhou B, Liu XY, Liu RF, et al: Exome sequencing of hepatitis $\mathrm{B}$ virus-associated hepatocellular carcinoma. Nat Genet 44 $1117-1121,2012$

49. Tilford CA and Siemers NO: Gene set enrichment analysis. Methods Mol Biol 563: 99-121, 2009.

50. Heitzmann D, Grahammer F, von Hahn T, Schmitt-Gräff A, Romeo E, Nitschke R, Gerlach U, Lang HJ, Verrey F, Barhanin J and Warth R: Heteromeric KCNE2/KCNQ1 potassium channels in the luminal membrane of gastric parietal cells. J Physiol 561: 547-557, 2004

51. Abbott GW and Roepke TK: KCNE2 and gastric cancer: Bench to bedside. Oncotarget 7: 17286-17287, 2016.

52. Zhang J, Zhao Z, Zu C, Hu H, Shen H, Zhang M and Wang J: Atrial natriuretic peptide modulates the proliferation of human gastric cancer cells via KCNQ1 expression. Oncol Lett 6 : 407-414, 2013
53. Liu X, Chen Z, Zhao X, Huang M, Wang C, Peng W, Yin J, Li J, $\mathrm{He}$ G, Li X and Zhu X: Effects of IGF2BP2, KCNQ1 and GCKR polymorphisms on clinical outcome in metastatic gastric cancer treated with EOF regimen. Pharmacogenomics 16: 959-970, 2015.

54. Than BL, Goos JA, Sarver AL, O'Sullivan MG, Rod A, Starr TK, Fijneman RJ, Meijer GA, Zhao L, Zhang Y, et al: The role of KCNQ1 in mouse and human gastrointestinal cancers. Oncogene 33: 3861-3868, 2014.

55. Wei Y, Wang T, Song H, Tian L, Lyu G, Zhao L and Xue Y: C-C motif chemokine 22 ligand (CCL22) concentrations in sera of gastric cancer patients are related to peritoneal metastasis and predict recurrence within one year after radical gastrectomy. J Surg Res 211: 266-278, 2017.

56. Zhang J, Zhou Y and Yang Y: CCR7 pathway induces epithelial-mesenchymal transition through up-regulation of Snail signaling in gastric cancer. Med Oncol 32: 467, 2015.

57. Wang WN, Chen Y, Zhang YD and Hu TH: The regulatory mechanism of CCR7 gene expression and its involvement in the metastasis and progression of gastric cancer. Tumour Biol 34: 1865-1871, 2013.

58. Wang W, Sun J, Li F, Li R, Gu Y, Liu C, Yang P, Zhu M, Chen L, Tian W, et al: A frequent somatic mutation in CD274 3'-UTR leads to protein over-expression in gastric cancer by disrupting miR-570 binding. Hum Mutat 33: 480-484, 2012.

59. Wei Y, Lin C, Li H, Xu Z, Wang J, Li R, Liu H, Zhang H, He H and $\mathrm{Xu}$ J: CXCL13 expression is prognostic and predictive for postoperative adjuvant chemotherapy benefit in patients with gastric cancer. Cancer Immunol Immunother 67: 261-269, 2018.

60. Ding Y, Shen J, Zhang G, Chen X, Wu J and Chen W: CD40 controls CXCR5-induced recruitment of myeloid-derived suppressor cells to gastric cancer. Oncotarget 6: 38901-38911, 2015.

61. Tian WY, Chen WC, Li R and Liu L: Markers CD40, VEGF, AKT, PI3K, and S100 correlate with tumor stage in gastric cancer. Onkologie 36: 26-31, 2013.

62. Dong Z, Fu S, Xu X, Yang Y, Du L, Li W, Kan S, Li Z, Zhang X, Wang L, et al: Leptin-mediated regulation of ICAM-1 is Rho/ROCK dependent and enhances gastric cancer cell migration. Br J Cancer 110: 1801-1810, 2014.

63. Wu CY, Wu MS, Chiang EP, Chen YJ, Chen CJ, Chi NH, Shih YT, Chen GH and Lin JT: Plasma matrix metalloproteinase-9 level is better than serum matrix metalloproteinase-9 level to predict gastric cancer evolution. Clin Cancer Res 13: 2054-2060, 2007.

64. Chen SW, Zhang Q, Xu ZF, Wang HP, Shi Y, Xu F, Zhang WJ Wang P and Li Y: HOXC6 promotes gastric cancer cell invasion by upregulating the expression of MMP9. Mol Med Rep 14 3261-3268, 2016

65. Xia Q, Bai QR, Dong M, Sun X, Zhang H, Cui J, Xi H, Hu XL, Shen $Q$ and Chen L: Interaction between gastric carcinoma cells and neural cells promotes perineural invasion by a pathway involving VCAM1. Dig Dis Sci 60: 3283-3292, 2015.

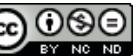

This work is licensed under a Creative Commons Attribution-NonCommercial-NoDerivatives 4.0 International (CC BY-NC-ND 4.0) License. 